\title{
OBESITY AND MALNUTRITION - RISK FACTORS IN PREDIAGNOSTIC AND LATER PHASES OF COLORECTAL CANCER
}

\author{
Racho S. RIBAROV ${ }^{1 凶}$ \\ ${ }^{1}$ Department of "Preventive Medicine“, Faculty of Public Health, Medical University - Sofia, Bulgaria \\ Received 27 Oct 2020, Accepted 19 Nov 2020 \\ https://doi.org/10.31688/ABMU.2020.55.4.13
}

\begin{abstract}
Obesity and malnutrition are subjected to comprehensive studies as risk factors in the pathogenesis of colorectal cancer (CRC). Nevertheless, the most recent scientific evidence revealed that a number of issues associated with risk impact processes remain to be clarified. The aim of the current review is to discuss the action mechanisms of obesity and malnutrition, their effect in the prediagnosis and later phases of CRC, with emphasis on concomitant oxidative stress. The relationship obesity-CRC is considered in three aspects: low-grade inflammation and role of adipocytokines, development of metabolic syndrome, lipid peroxidation and oxidative stress. Malnutrition effect is discussed in two aspects metabolic dysregulation and oxidative stress. Data are presented on the specificity of action of the two risk factors referring to the periods of disease course. Oxidative stress is a characteristic indicator of obesity, malnutrition and CRC. This fact enables us to recommend its inclusion together with nutritional status indicators in CRC screening.
\end{abstract}

Keywords: colorectal cancer, obesity, malnutrition, oxidative stress.

\section{Résumé}

Obésité et malnutrition - facteurs de risque dans les phases prédiagnostique et ultérieure du cancer colorectal

L'obésité et la malnutrition en tant que facteurs de risque d'initiation et de progression du cancer colorectal (CCR) font l'objet d'études approfondies. Cependant les dernières évidences scientifiques démontrent divers problèmes non clarifiés associés aux processus d'impact des risques. L'objectif de la revue actuelle était de présenter d'information sur les mécanismes d'action de l'obésité et de la malnutrition, leur effet aux phases prédiagnostique et ultérieure du treatment du CCR en mettant l'accent sur le stress oxydatif. La relation entre l'obésité et le CCR est considérée sous trois aspects: inflammation de faible intensité et rôle des adipocytokines; développement du syndrome métabolique; peroxydation lipidique et stress oxydatif. L'effet de la malnutrition est discuté sous deux aspects: la dereglement métabolique et le stress oxydatif. Des données sur la spécificité d'action des deux facteurs de risque sont présentées se référant aux périodes du prédiagnostic et du treatment de la maladie. Le stress oxydatif est un indicateur caractéristique de l'obésité, de la malnutrition et du CCR. Ce fait nous permet de 


\section{List of abbreviations}

$\mathrm{CRC}=$ Colorectal cancer

$\mathrm{CRP}=$ C-reactive protein

ROS $=$ Reactive oxygen species

$\mathrm{BMI}=$ Body mass index

$\mathrm{BWL}=$ Body weight loss

$\mathrm{RR}=$ Relative risk

$\mathrm{IL}=$ Interleukin

TNF $=$ Tumor necrosis factor

$\mathrm{IGF}=$ Insulin growth factor

IGFBP = IGF-binding protein

$\mathrm{MDA}=$ Malondialdehyde

4-HNE = 4-hydroxy-2-nonenal

$\mathrm{SOD}=$ Superoxide dismutase

\section{INTRODUCTION}

The systematic growth of colorectal cancer (CRC) prevalence in the last years ranks it third by prevalence rate and second by mortality rate among all malignant diseases ${ }^{1}$. Global 2018 data showing 18.1 million new cases and 9.6 million deaths ${ }^{2}$ are a challenge to current medical science.

Numerous studies responding to this challenge were and currently are focused on clarifying the role of the constantly increasing number of various risk factors, including malnutrition and obesity. Most studies, though, treated those risk factors separately when explaining their role in CRC development. Scientific studies on the individual stages of disease progression, from its onset to the clinical outcome, have found various pathological levels of nutritional status. Although there are differences in the pathophysiology of obesity and malnutrition, many of the indicators are inherent in both states, including anthropometric, immunological and biochemical markers with specific complexity and extent of their manifestation. One of them, for example, is the anthropometric indicator body mass index (BMI) which has been the subject of various studies and discussions as a marker of the nutrition - CRC relationship ${ }^{3-5}$.

The immunological indicators provide information about the production of various pro-inflammatory cytokines associated with inflammatory processes, with particular role in the association between malnutrition and obesity, on one side, and CRC, on the other side ${ }^{6-7}$.

Biochemical indicators characterizing oxidative stress, concomitant with both obesity and malnutrition, have been used in various studies providing data for epigenetic changes in the gene expression in the colorectal cell under reactive oxygen species (ROS) impact ${ }^{8-10}$.

Certain experimental and epidemiological studies supported the relationship between obesity as recommander son inclusion ensemble avec des indicateurs d'état nutritionnel dans les études de dépistage du CCR.

Mots-clés: cancer colorectal, obésité, malnutrition, stress oxydatif.

a risk factor and the initiation and progression of $\mathrm{CRC}^{11-12}$. From this point of view, the data were unidirectional, characterizing mainly the pre-diagnostic phase of the disease. Nevertheless, studies on the nutritional status of patients in the post-diagnostic period showed significant effect on life quality, causing loss of appetite, body weight loss (BWL), malnutrition, radically differing from those of obesity status $^{5-7}$. Those results outlined the necessity for furthermore comprehensive studies of the yet unclear "unique" (in the sense of hard-to-explain) relationship between BMI and CRC. This issue is currently being researched through various approaches in disease prevention and treatment process. How could the differing pathophysiological states like obesity and malnutrition be included in the disease initiation and progress?

The current scientific publications provide relatively few and even scarce data on the effect of obesity in the treatment course compared to the richer information on malnutrition as a risk factor for this period. The necessity to clarify the processes along which obesity and malnutrition, concomitant to CRC initiation and progress, could contribute to the effectiveness of the treatment and improve the precision of patients' survival predictions is clearly outlined.

The aim of the current review is to focus the attention on CRC patients' nutritional status, to present an analysis of the scientific information about the mechanisms of action of obesity and malnutrition during the individual periods of pre-diagnostic and later phases of CRC, with emphasis on concomitant oxidative stress. The analysis covered scientific medical journals in the field of oncology, nutrition, gastro-enterology and surgery, published lately. The publications were selected using the Scopus information system, with the following keywords: colorectal cancer, obesity, malnutrition, BMI, oxidative stress, survival. The results of the analysis will be presented 
in two chapters as follows: obesity - CRC, malnutrition - CRC, with an accent on oxidative stress concomitant in both pathophysiological states.

\section{Obesity - CRC}

There are numerous studies on the role of nutrition, identifying obesity as a risk factor for the development of malignant diseases ${ }^{13,14}$, and CRC has been in the focus of scientific studies for decades ${ }^{5,9}$.

Certain meta-analyses on numerous epidemiological studies of the obesity - CRC relationship present data confirming this relationship. Moghaddam et al, in 2007, analyzing 31 individual surveys covering 70,000 events, have proven a direct and indirect relationship between obesity and CRC, underlying, though at lower significance level, those presented in papers published before ${ }^{15}$.

As a result of meta-analyses, quantitative expressions of this relationship have been elaborated, proving that each 5-fold increase of BMI increased the risk for $\mathrm{CRC}$ development in both men $(\mathrm{RR}=1.30)$ and women $(R R=1.12)$, but only men's data are highly significant $(\mathrm{p}<0.001)_{16}$. In this study, the authors proved that a $10 \mathrm{~cm}$ increase of the waist circumference and 0.1 increase of the waist/hip ratio increase CRC risk for both men and women ${ }^{16}$.

The exact biochemical action mechanism of obesity in initiating CRC is not yet clarified, but the accumulated evidence from pre-clinical and clinical studies on tumour-promotive effect supported the different aspects of this relationship. The greater part of the reviewed information enabled the differentiation, though conditionally to a certain extent, the discussed possible mechanisms of obesity impact. They could be analyzed in the following aspects:

- Obesity as a low-grade inflammation - role of adipocytokines.

- Obesity as a risk of developing metabolic syndrome.

- Obesity and oxidative stress - a risk factor for CRC.

It is known that fat tissue is an active endocrine organ, synthesizing a big number of bioactive proteins - adipokines. Increased pro-inflammatory adipokines are observed in obesity, such as interleukin-6 (IL-6), tumour necrosis factor (TNF) and leptin, and the level of adiponectin, which is an anti-inflammatory adipokine, is lowered. The elevated levels of pro-inflammatory cytokines explain obesity as a low-grade inflammatory disease, with insulin resistance. On the other hand, high cytokines levels explain the relationship with the tumoral genesis through angiogenesis activation $^{6,13}$. The diagnostic and prognostic value of serum IL-6 in CRC has been proven. The elevated levels are associated with bad prognosis for disease progressing and, respectively, for survival. A correlation has been established between TNF- $\alpha$ expression in parallel with the increased value of $\mathrm{BMI}^{17}$. It is logical to suppose that the long-term inflammatory process could initiate and accelerate tumoral genesis.

The second aspect explaining the relationship between obesity and CRC is associated with the metabolic syndrome that usually accompanies obesity and overweight. Being a chronic inflammatory process, metabolic syndrome is also a carcinogenesis-predisposing state. Obesity, and particularly abdominal obesity, is associated with hyperinsulinemia and insulin resistance. There is lot of evidence emphasizing high insulin levels as a risk factor for certain malignant diseases ${ }^{3,17}$.

Epidemiological studies have proven the involvement of various indicators characterizing the relationship between obesity and carcinogenesis, such as high concentration of the serum C-reactive protein (CRP), insulin growth factor-1 (IGF-1), IGF-binding protein-3 (IGFBP-3) for which there is a respective relationship with $\mathrm{BMI}^{6,7}$. Giovannucci et al. proved that elevated levels of IGF-1 and decreased levels of IGFBP-3 were independently related to increased CRC development. Giovannucci et al confirmed that the risk was 4-times higher at IGFBP-3 decrease, while the elevated level of IGF-1 caused twofold risk increase ${ }^{18,19}$. Not all recent studies on the role of IGF-1, IGF-1I in cancer were unidirectional in this aspect - some of them confirm $\mathrm{it}^{20}$, and others did not find similar relationships ${ }^{21}$.

The role of the free radicals in the initiation and progressing of carcinogenesis, as well as the involvement of oxidative stress in obesity, have been confirmed. The disturbed oxidant/antioxidant balance causes oxidative stress, which is a risk factor for deregulation of multiple pathways, associated with cellular proliferation, differentiation, survival and apoptosis. There are numerous scientific publications confirming the role of oxidative stress in the initiation and progression of CRC, outlining the importance of total antioxidant capacity in prevention and treatment processes ${ }^{22-24}$. Studies on humans and laboratory animals conducted yet in the beginning of the past century have found that fat accumulation correlated with oxidative stress, causing dysregulated adipocytokines production engaged in the pathogenesis of obesity-associated metabolic syndrome ${ }^{25}$.

Obesity as a chronic low-grade inflammation is accompanied by oxidative stress, which causes fatty-acid peroxidation, leading to the formation of strong toxic and mutagenic compounds, directly associated with high proliferative $\mathrm{CRC}^{6,26}$.

Nowadays, metabolites produced by fat tissue in the process of lipid peroxidation, with pro-inflammatory and carcinogenic characteristics, have been identified. Those that were studied more profoundly 
were malondialdehyde (MDA) and 4-hydroxy-2-nonenal (4-HNE). MDA was associated more with its mutagenic activity and tumourogenic properties, while 4-HNE had mainly toxic properties, causing deregulation of various pathways ${ }^{6}$.

Although the relationship between obesity and CRC is not yet fully clarified, the puzzle is constantly completing by adding the oxidative stress factor to the already discussed factors - metabolic syndrome, insulin resistance and adipocytokines activity.

There are numerous studies proving the relationship between obesity and CRC initiation, but the data about its effect on survival and clinical outcome after diagnosis and surgical treatment of patients with CRC are scarce. Possibly, the lower interest in obesity in the post-surgery treatment of CRC is due to the scarcer number of patients with obesity in this period of the disease progress and treatment, when the prevailing state is malnutrition.

\section{Malnutrition - CRC}

Malnutrition is a frequently encountered health issue, accompanying several diseases as well as the life of risk population groups from regions of least developing countries. Malnutrition is observed also in some developed countries, characterized by certain nutrient deficiencies, altering the regional disease profile from infectious to chronic degenerative diseases ${ }^{27}$. The World Health Organization defined malnutrition as "the cellular imbalance between supply of nutrients and energy, along with the body's demand for them to ensure growth, maintenance, and specific functions ${ }^{\text {" } 28}$.

Malignant diseases are usually accompanied by BWL, decreased physical activity, malnutrition and disturbed lifestyle. The role of malnutrition in CRC development will be presented in two aspects: metabolic changes and effect of oxidative stress.

Malnutrition is a serious issue in cancer patients. Its incidence is different depending on cancer type, but is always associated with a bad prognosis ${ }^{29,30}$. In all patients with cancer treated surgically, the extent of malnutrition progression must be assessed. Malnutrition is a serious risk factor particularly for CRC, associated usually with long post-operative hospitalization, complications and increased post-operative 30-day mortality rate ${ }^{31}$. BWL of more than $10 \%$ during the pre-diagnosis - post-diagnosis period of disease course correlates with poor overall survival ${ }^{5,7}$. A study covering 13,239 patients with CRC, conducted in the period 2010-2014, showed that in patients with a small risk for malnutrition, the aggravations and concomitant morbidity were less ${ }^{32}$, having a better survival.

The most frequently used indicators for malnutrition assessment are hypo-albuminemia, BWL and
BMI. The comparative assessment of the significance of those indicators identifies low serum albumin as a more precise marker for prediction of post-operative mortality rate among CRC patients, while its pre-operative values are more important for the assessment of nutritional status associated with surgical risk ${ }^{31}$. The traditionally low level of serum albumin is associated with malnutrition and necessity of active nutritional intervention. In cancer patients, though, its power as a marker in this aspect can be reduced because of its possible inhibition by the elevated inflammatory cytokines levels - specific characteristic for those diseases. That is why the combined use of the three indicators is recommended.

Protein metabolism in most cancer events is disturbed, with evidenced proteolysis and reduced protein synthesis, causing loss of muscle mass and muscle power. The proteolytic pathways, as ATP ubiquitin-dependent proteolytic pathway, calcium/calpain pathway and lysosomal cathepsins are involved in the proteolysis process, early marker for tumour growth, much before its clinical manifestation ${ }^{33}$. The reduced muscle strength is due to the disintegration of the myofibrillar components, inflammatory cytokines, stress hormones and mitochondrial dysfunction ${ }^{34}$. The recommendations for overcoming protein catabolism in cancer include a high-protein diet, containing essential amino acids and the non-essential amino acid glutamine, which is observed in lower levels in cancer patients. Glutamine supplementation of patients with CRC during chemotherapy showed significant reduction of mucositis and ulceration of gastrointestinal mucosa $\mathrm{a}^{35}$. Other studies using supplements containing a combination of three amino acids - leucine, glutamine and arginine - evidenced body mass gain ${ }^{36}$. The presented data are similar with the recommendations for increased high biological value proteins intake. Amino acids, besides being building elements in protein synthesis, are involved also in the regulation of various biochemical reactions and processes: cell signaling pathways, antioxidant protection, acid-base balance, gene expression, immune function, energy and nutrient metabolism and others.

The disturbed carbohydrate metabolism in cancer, characterized with high glycolysis level, is well documented and glucose demands are satisfied through glucogenesis in the liver, using lactate produced by cancer cells ${ }^{7,37}$. The significant role of carbohydrate metabolism is explained by the huge energy demand of the cancer cell. The data for the role of carbohydrate metabolism at CRC differ - some studies proved an association between the markers of glucose metabolism and CRC progressing ${ }^{17}$, while other surveys did not reveal similar relationships ${ }^{38}$. In this aspect, there is another issue to be explained - following the 
recommendations for restriction of the dietary energy intake at concomitant CRC malnutrition.

Lipid metabolism disorders in patients with cancer accompanied by malnutrition are expressed in higher lipolysis and fat oxidation rates. In patients with cancer-associated cachexia, higher levels of free fatty acids are found due to lipolysis activated by hormones, pro-inflammatory cytokines, and lipid-mobilizing factor $^{39}$. Petruzzelli and Wagner communicated interesting evidence on lipid metabolism during cancer-associated cachexia with transformation of white adipose tissue cells to brown adipose tissue-like cells, a process associated with imbalance in homeostasis and catabolic wasting ${ }^{40}$.

Frequently observed cachexia in CRC patients is not only a sequence of malnutrition, but disordered protein, carbohydrate and lipid metabolism with increased energy demands. It has been confirmed that some compounds with signal functions (TNF- $\alpha$, IL-6) are involved in cancer-associated cachexia ${ }^{41}$ when the level of insulin-like growth factor-1 is elevated, causing insulin resistance ${ }^{37}$. The changes in the metabolism of macronutrients in CRC, causing malnutrition, are also due to the interrelation of tumour factors and host-tumour factors ${ }^{33}$.

Malnutrition impairs the body's natural anti-oxidative defenses and may alter the integrity of cell membranes and the metabolic pathways in cells. The studies in this aspect are unidirectional, proving excessive production of free radicals and direct relationship between the immune system and malnutrition-concomitant oxidative stress in the etiology of $\mathrm{CRC}^{42,43}$.

The main indicators used for the assessment of oxidative stress in CRC accompanied by malnutrition are: MDA - product of lipid peroxidation engaged in malnutrition pathophysiology; superoxide dismutase (SOD) - enzyme, acting in the first line of the antioxidant protection against the effect of free radicals; vitamin E - powerful antioxidant interrupting the lipid peroxidation chain; zinc - essential for the function of metal-enzymes with antioxidant activity, which deficiency is associated with malnutrition ${ }^{27}$. The assessment of the oxidative stress will assist the choice of a correct approach to dietary intervention in CRC accompanied by malnutrition.

The current scientific literature presents large information about the effect of various antioxidants on the progressing of $\mathrm{CRC}$, but not all publications have confirmed their positive effect ${ }^{9,23}$, thus demanding additional studies.

\section{Conclusions}

The analysis of current scientific literature reveals that obesity and malnutrition, as border forms of nutritional status, have a definite role during the different periods of CRC progression. While obesity is one of the risk factors for disease initiation, malnutrition acts mainly after diagnosis and, more particularly, after surgical treatment. This distribution of risk factors in the period of initiation and progression of the disease is important for the identification of effective preventive and therapeutic approaches.

The scientific evidence enables the suggestion that not obesity and malnutrition per se, but concomitant immune response and inflammation promote CRC.

Oxidative stress is a characteristic indicator for both obesity and malnutrition, and for CRC is the first stage of carcinogenesis. This uniting role of oxidative stress enables the recommendation to incorporate its indicators together with nutritional status indicators in CRC-related screening studies.

\section{Author Contributions:}

R.S.R. conceived the original draft preparation and was responsible for conception and design of the review. R.S.R. was responsible for the data acquisition, collection and assembly of the articles/published data, and their inclusion and interpretation in this review.

\section{Compliance with Ethics Requirements:}

"The author declares no conflict of interest regarding this article."

„No funding was requested for this study"

\section{Acknowledgements:}

None

\section{References}

1. Siegel RL, Miller KD, Jemal A. Cancer statistics. CA. Cancer J Clin. 2015;65(1):5-29.

2. Bray F, Ferlay J, Soerjomataram I, Siegel RL, Torre LA, Jemal A. Global cancer statistics 2018: GLOBOCAN estimates of incidence and mortality worldwide for 36 cancers in 185 countries. CA. Cancer J. Clin. 2018;68:394-424.

3. Kwon H, Pessin JE. Adipokines mediate inflammation and insulin resistance. Frontiers in Endocrinology. 2013;4, article 71.

4. Neumann K, Mahmud SM, McKay A, et al. Is obesity associated with advanced stage or grade of colon cancer? Can J Surg. 2015;58:140-142.

5. Shahjehan F, Merchea A, Cochuyt JJ, Colibaseanu DT, Kasi PM. Body mass index and long-term outcomes in patients with colorectal cancer. Front Oncol 2018;17(8):620.

6. Martinez-Useros J, Garcia-Foncillas J. Obesity and colorectal cancer: molecular features of adipose tissue. Journal of Translational Medicine 2016;14:21.

7. Kim DH. Nutritional issues in patients with cancer. Intest Res. 2019;17(4):455-462. 
8. Zhang R, Kang KA, Kim KC, et al. Oxidative stress causes epigenetic alteration of CDX1 expression in colorectal cancer cells. Gene. 2013; 524(2):214-219.

9. Chalons $\mathrm{P}$, Courtaut F, Limagne E, et al. Red wine extract disrupts Th17 lymphocyte differentiation in a colorectal cancer context. Molecular Nutrition. 2020;64(11):1901286.

10. Kang KA, Kim KC, Bae SC, Hyun JW. Oxidative stress induces proliferation of colorectal cancer cells by inhibiting RUNX3 and activating the Akt signaling pathway. International Journal of Oncology. 2013;43(5):1511-1516.

11. World Cancer Research Fund/ American Institute for Cancer Research. Food, Nutrition, Physical Activity, and the Prevention of Cancer: a Global Perspective. Washington DC; AICR, 2007.

12. Nagata N, Sakamoto K, Arai T, et al. Visceral abdominal fat measurement by computed tomography is associated with an increased risk of colorectal adenoma. Int $J$ Cancer. 2014;135(10):2273-81.

13. Nigro E, Scudiero O, Monaco ML, et al. New insight into adiponectin role in obesity and obesity-related diseases. Biomed Res Int. 2014; 2014:658913.

14. Virizuela JA, Camblor-Alvarez M, Luengo-Perez LM, et al. Nutritional support and parenteral nutrition in cancer patients: an expert consensus report. Clin Transl Oncol. 2018;20:619-629.

15. Moghaddam AA, Woodward M, Huxley R. Obesity and risk of colorectal cancer: a meta-analysis of 31 studies with 70,000 events. Cancer Epidemiol Biomarkers Prev. 2007;16(12):2533-2547.

16. Aykan NF, Artak M, Ozatli T. Body mass index and colorectal cancer. In: Body Mass Index and Health. Onal AE, IntechOpen, 2018. Available at: www.intechopen. $\mathrm{com} /$ books/body-mass-index-and-health/body-mass-index-and-colorectal-cancer.

17. Xu J, Ye Y, Wu H, et al. Association between markers of glucose metabolism and risk of colorectal cancer. BMJ Open. 2016;6:e011430.

18. Giovannucci E. Insulin-like growth factor-1 and binding protein-3 and risk of cancer. Horm Res. 1999;51,S3: 34-41.

19. Giovannucci E, Pollak MN, Piatz EA, et al. A prospective study of plasma insulin-like growth factor-1 and binding protein-3 and risk of colorectal neoplasia in women. Cancer Epidemiol Biomarkers Prev. 2000;9(4):345-349.

20. Rahmani J, Varkaneh K, Clarc C, et al. The influence of fasting and energy restricting diets on IGF-I levels in humans: a systematic review and meta-analysis. Ageing Res. Rev. 2019;53:100910.

21. Suzuki S, Kojima M, Tokudome S, et al. Insulin-like growth factor (IGF)-I, IGF-II, IGF binding protein-3, and risk of colorectal cancer: A nested case-control study in Japan Collaborative Cohort study. Asian Pac. J. Cancer Prev. 2009;10:45-49.

22. La Vecchia C, Decarli A, Serafini M, et al. Dietary total antioxidant capacity and colorectal cancer: A large-control study in Italy. International J of Cancer. 2013;133:1447-1452.

23. Pais R, Dumitrascu DL. Do antioxidants prevent colorectal cancer? A meta-analysis. Rom J Intern Med. 2013;51(3-4):152163.

24. Lin S, Zamyatnin AAJ, Werner J, Bazhin AV. Reactive oxygen species and colorectal cancer. J Cell Physiol. 2018;233:5119-5132.

25. Furukawa S, Fujita T, Shimabukuro M, et al. Increased oxidative stress in obesity and its impact on metabolic syndrome. J Clin Invest. 2004;114(12):1752-1761.
26. Vermorken AJ, Zhu J, Andres E. Obesity and colorectal cancer risk: the role of oxidative stress. Gut. 2014;63(3):529530.

27. Mastorci F, Vassalle C, Chatzianagnostou K, et al. Undernutrition and overnutrition burden for diseases in developing countries: the role of oxidative stress biomarkers to assess disease risk and interventional strategies. Antioxidants. 2017;6(2):41.

28. WHO [Internet]. Malnutrition-The Global Picture. Geneva: World Health Organization 2002 [Cited 20 Sep 2010]. Available from: http://www.who.int/home-page.

29. Gupta D, Vashi PG, Lammersfeld CA, Braun DP. Role of nutritional status in predicting the length of stay in hospital: a systematic review of the epidemiological literature. Ann Nutr Metab. 2011;59:96-106.

30. Marshall KM, Loeliger J, Nolte L, Kelaart A, Kiss NK. Prevalence of malnutrition and impact on clinical outcomes in cancer services: a comparison of two time points. Clin Nutr. 2019;38:644-651.

31. Hu W-H, Cajas-Monson LC, Eisenstein S, Parry L, Cosman B, Ramamoorthy S. Preoperative malnutrition assessments as predictors of postoperative mortality and morbidity in colorectal cancer: an analysis of ACS-NSQIP. Nutr J. 2015;14:91

32. Meissner C, Fahlke I, Otto R, et al. The risk of malnutrition in patients with a colon carcinoma: An analysis of $13239 \mathrm{pa}-$ tients. Journal of Clinical Oncology. 2017;35(15):suppl.e15057.

33. Donohoe CL, Ryan AM, Reynolds J. Cancer cachexia: mechanisms and clinical implications. Gastroenterol Res Pract. 2011;2011:601434.

34. Cohen S, Nathan JA, Goldberg AL. Muscle wasting in disease: molecular mechanisms and promising therapies. Nat Rev Drug Discov. 2015;14:58-74.

35. Decker-Baumann C, Buhl K, Frohmuller S, von Herbay A, Dueck M, Schlag PM. Reduction of chemotherapy-induced side-effects by parenteral glutamine supplementation in patients with metastatic colorectal cancer. Eur J Cancer. 1999;35:202-207.

36. Dev R, Wong A, Hui D, Bruera E. The evolving approach to management of cancer cachexia. Oncology Journal. 2017;31(1):23-32.

37. Porporato PE. Understanding cachexia as a cancer metabolism syndrome. Oncogenesis. 2016;5:e200.

38. Kabat GC, Kim MY, Strickler HD, et al. A longitudinal study of serum insulin and glucose levels in relation to colorectal cancer risk among postmenopausal women. $\mathrm{Br} J$ Cancer. 2012;106:227-232.

39. Tsoli M, Swarbrick MM, Robertson GR. Lipolytic and thermogenic depletion of adipose tissue in cancer cachexia. Semin Cell Dev Biol. 2016;54:68-81.

40. Petrruzzelli M, Wagner EF. Mechanisms of metabolic dysfunction in cancer-associated cachexia. Genes Dev. 2016;30:489-501.

41. Laine A, Iyengar P, Pandita TK. The role of inflammatory pathways in cancer-associated cachexia and radiation resistance. Mol Cancer Res. 2013;11:967-972.

42. Mandal P. Potential biomarkers associated with oxidative stress for risk assessment of colorectal cancer. Arch Pharmacol. 2017;390(5):557-565.

43. Castjon M, Plaza A, Martinez-Romero J, Fernandez-Marcos PJ, de Cabo R, Diaz-Ruiz A. Energy restriction and colorectal cancer: a call for additional research. Nutrients. 2020;12(1):114. 